\title{
Petroclival Region
}

National Cancer Institute

\section{Source}

National Cancer Institute. Petroclival Region. NCI Thesaurus. Code C62644.

The area between the clivus and the petrous portion of the temporal bone. 